\title{
Human Safety in Veterinary Microbiology Laboratory
}

\author{
Shankar B. P., H. S. Madhusudhan ${ }^{1}$ and Harish. D. B ${ }^{2}$ \\ Dept. of Veterinary Pathology, \\ Veterinary College, KVAFSU, Hebbal, Bangalore-24. India.
}

\begin{abstract}
Laboratory work should be carried out with a minimum of risk to the health of the staff working in laboratory. This requires careful consideration of the risks involved in a particular procedure, followed by appropriate measures to minimise the risk of human disease. This concerned exclusively with risks from infectious agents, but physical and chemical injuries in microbiology laboratories must also be prevented. Risks from infection are reduced by good laboratory techniques and secured facilities which aid in the containment of pathogens. It is important to understand that containment of pathogens can be used for preventing disease in humans and animals. Often the same methods of containment are used for both preventing laboratory-acquired infection in humans and for preventing escape of pathogens that could cause an outbreak of animal disease. Although the methods, techniques and facilities required may be the same, the list of pathogens and categorization into levels of risk will differ depending on whether it is human or animal disease control that is the primary objective. Existing national and international reference laboratories have considerable experience in the operation of safe working practices and provision of appropriate facilities. When new laboratories are being established, it would be prudent to seek advice from the competent authorities at established institutes and it is important to comply with legislative requirements.
\end{abstract}

Keywords: Safety, Laboratory, Microbiology, Veterinary, Pathogen, Infectious Material, Diagnosis, Physical Hazard, Chemical Hazard.

\section{Assessment of Risk from Pathogens}

It is necessary first to assess the risk from a pathogen, so that it can be assigned to a risk group. Work with it can then be assigned to an appropriate containment category. To assess the risk to humans from a particular pathogen it is necessary to know whether infection with that organism can cause death, disease or inconvenience to the people working with it and whether it could then spread to cause disease in the general human population. To assess this risk it is necessary to know the epidemiological background of the organism and also such attributes of the organism as infectivity for humans, stability in the environment, ability to infect by different routes and susceptibility to specific treatments or prophylaxis. It is relatively easy to obtain this information when working with a known pathogen, but the problem is more complex in a diagnostic laboratory receiving clinical material that may be infected with a variety of unknown pathogens which could be extremely hazardous to human health.

Some of the considerations to take into account when evaluating risk are:

1. Known occurrence of human infection with the organism or related organisms with similar characteristics, any history of laboratory-acquired infection, infective dose and disease severity. Production of toxins or allergens.

2. The volume of culture to be handled and the concentration of the organism likely to be present.

3. The origin of the sample, e.g. samples from wildlife species may contain human pathogens not normally encountered.

4. The history of the isolate being handled. Pathogens on primary isolation or of low passage level are often more dangerous than pathogens of high passage level.

5. The possibility of aerosol formation should be especially taken into consideration when handling fluid samples, e.g. homogenization and centrifugation.

6 . The threat that the organism may pose to foodproducing or companion animals or to wildlife, irrespective of the threat to laboratory personnel. Additional precautions for handling and storage are required for animal disease agents from foreign countries.

1. Dept. of Animal Nutrition,

2. Dept. of Poultry sciences, 
Human Safety in Veterinary Microbiology Laboratory

7. The physical state of the employees. For example, in the case of pregnancy, immunodeficiency or allergy, special precautions may be required. Sometimes certain individuals have to be excluded from particular types of work that would be especially hazardous to them.

8. A higher level of risk may arise when agents such as Brucella or Mycobacteria are inoculated into animals. To evaluate the impact of animal inoculation, a risk assessment should be conducted and the following factors should be considered:

a) Host species versus inoculated species.

b) Strain/treatment and concentration of the inoculum.

c) Route of inoculation.

d) Animal housing.

e) Types of sampling during the experiment.

\section{Grouping of Microorganisms}

The considerations outlined above have been used by several National Authorities to designate microorganisms into four hazard groups representing increasing hazards to human health. Such categorization of pathogens makes no allowance for people who are particularly susceptible, for example due to pre-existing disease, a compromised immune system or pregnancy. The four groups may be summarized thus:

Group 1 - Organisms that are unlikely to cause human disease.

Group 2 - Organisms that may cause human disease but are unlikely to be spread in the community and for which effective prophylaxis and treatment are available. Group 3 - Organisms that can cause severe human disease and may spread in the community but for which there is usually effective prophylaxis and treatment.

Group 4 - Organisms that cause severe human disease, may represent a high risk of spread in the community and for which there is usually no effective prophylaxis or treatment.

Infectious organisms that might be encountered in laboratory work have been assigned to Hazard Groups 1-4 by authorities in several countries. Bovine spongiform encephalopathy (BSE) has been placed in Hazard Group 3 by the European Union. Also, some very serious Group 4 agents, including Hendra and Nipah, have been isolated from diagnostic specimens in veterinary laboratories.

Group 2 Viruses: Influenza viruses types A, B, C; Newcastle disease virus; Orf (parapox virus)

Bacteria: Alcaligenes spp.; Arizona spp.; Campylobacter spp.; Chlamydia psittaci (nonavian); Clostridium tetani; Clostridium botulinum;
Corynebacterium spp.; Erysipelothrix rhusiopathiae; Escherichia coli; Haemophilus spp.; Leptospira spp.; Listeria monocytogenes; Moraxella spp.; Mycobacterium avium; Pasteurella spp.; Proteus spp.; Pseudomonas spp.; Salmonella spp.; Staphylococcus spp.; Yersinia enterocolitica; Yersinia pseudotuberculosis Fungi: Aspergillus fumigatus; Microsporum spp.; Trichophyton spp.

Group 3 Viruses: Rabies virus; Equine encephalomyelitis virus (Eastern, Western and Venezuelan); Japanese B encephalitis virus; Louping ill virus

Bacteria: Bacillus anthracis; Burkholderia mallei (Pseudomonas mallei); Brucella spp.; Chlamydia psittaci (avian strains only); Coxiella burnetti; Mycobacterium bovis.

\section{Requirements for work with infectious agents}

A. Known pathogens: Having decided the risk level of certain work it is then possible to decide the appropriate 'containment level' that is needed to minimise the risk of human disease. The containment level is defined by a combination of the physical facilities and working practices employed. Organisms of the four risk groups indicated above may be placed into containment levels appropriate for safe working. Laboratories usually appoint a safety officer, responsible for ensuring that microorganisms are handled at the appropriate containment level. They should have sufficient expertise and be of sufficient seniority to oversee and advise on all safety matters. In large organisations with a network of laboratories, it is appropriate to appoint a central safety officer to advise on and coordinate safety matters of a corporate nature which are implemented by local laboratory safety officers at each site. The working methods for a particular procedure or work station should be written out and readily available. Staff must be fully trained and fully aware of any health risks associated with their work and in procedures for reporting incidents or accidents. Staff should also be given a medical card indicating pathogens to which they might be exposed. In some cases, staff can be specially vaccinated to give additional protection, e.g. when working with the rabies virus; this should also be recorded on the medical card. Such information is useful for a medical practitioner in the event of illness occurring. Regular medical examinations are recommended for employees those who are working with the organisms that cause certain serious human diseases such as brucellosis and tuberculosis.

Much information is available on containment of pathogens and sophisticated apparatus and buildings may be constructed for containment of the more 
Human Safety in Veterinary Microbiology Laboratory

hazardous organisms as required by the guidelines, standards and regulations of each country. The requirements depend on the containment required from the most basic to the highest level. The essential requirements for any work with infectious agents are as follows:

1. The laboratory should be easy to clean with surfaces that are impervious to water and resistant to chemicals. There must be a wash-hand basin and emergency shower, including an eye bath, in each laboratory suite as appropriate for the chemicals and other hazards present. Procedures must be established for frequent cleaning and disinfection during and at the end of the work period.

2. Personnel access to the laboratory should be restricted.

3. Protective clothing, including gloves, masks, eye shields, and oro-nasal respirators, as appropriate, must be worn in the laboratory and removed when leaving the laboratory.

4. The laboratory door should be closed when work is in progress and ventilation should be provided by extracting air from the room. (Where biohazard cabinets are used, care must be taken to balance ventilation systems.).

5. Food or drink must not be stored or consumed in laboratories.

6. Smoking or application of cosmetics must not take place in the laboratory.

7. Pipetting must not be done by mouth.

8. Care must be taken to minimise the production of aerosols.

9. Emergency response plans should be developed to deal with biohazard spills. Some of the items addressed in the plans should include having effective disinfectant available for cleaning spills, removal of and decontamination of contaminated protective clothing, washing of hands, and cleaning and disinfection of bench tops.

10. Used laboratory glassware and other materials must be stored safely before disinfection. Materials for disposal must be transported without spillage in strong containers. Waste material should be autoclaved, incinerated or otherwise made safe before disposal. Reusable material must be decontaminated by appropriate means.

11. No infectious material shall be discarded down laboratory sinks or any other drain.

12. Any accidents or incidents must be recorded and reported to the safety officer.

Containment level for Group 2 pathogens, in addition to the points given above, a microbiological safety cabinet should be used but can be operated in the open front mode (Class I cabinet). A Class II cabinet may also be used when there is potential for generating aerosols or when handling large quantities of culture or where there is a real need to protect the biological product. Appropriate signs are required at all entry doors to indicate the hazard present and the name and telephone number of the person(s) responsible. Emergency protocols should be posted within the laboratory to advise personnel of procedures to follow in case of a pathogen spill or the need to evacuate the laboratory in the event of a fire or other emergency.

Containment level for Group 3 pathogens, it is advisable that the laboratory be in an isolated location; access should be limited to qualified level 3 staff. Emergency protocols should be posted within the laboratory to advice personnel of procedures to follow in case of a pathogen spill or the need to evacuate the laboratory in the event of a fire. OIE containment level for group 3 pathogens surpasses biosafety level-3 (BSL-3) guidelines as outlined by the United States Department of Health and Human Services (DHHS) and the United States Department of Agriculture (USDA).

In addition to the previous requirements, the laboratory must be under negative pressure and the pressure differentials should be monitored; a procedure should be developed to provide an alarm if there is a problem. A ventilation system is required that removes air from the laboratory through a high efficiency particulate air (HEPA) filter. HEPA filters most be verified regularly; this would include HEPA filters in biosafety cabinets and on room and equipment exhausts. The laboratory should be sealable for fumigation and contain an airlock entry. There is a requirement to treat effluent depending on the pathogen. Safety cabinets of Class I, II or III must be provided. It may be necessary for staff to shower on exit from the laboratory and they must wear dedicated laboratory clothing that is left in the laboratory before leaving the building.

Containment level for Group 4 pathogens, the most stringent precautions are required including access to the building through a system of air locks and the building being maintained under negative air pressure. Inlet air to the laboratory must be filtered through a single HEPA filter and extracted air through double HEPA filters. All work with infective materials must be conducted in a Class III cabinet or in a Class Il cabinet in conjunction with the use of positivepressure personnel suits. All sewage from the laboratory, laboratory effluent and autoclave drain effluent must be treated by appropriate means to ensure that all infectious material is destroyed before entering the public sewerage system. Staff must 
Human Safety in Veterinary Microbiology Laboratory

shower and change their clothing before leaving the building. Other precautions as described for Group 3 would also apply. The use of one-piece positivepressure suits is now an internationally accepted way of providing additional protection at level 4 .

B. Diagnostic specimens : Veterinary diagnostic centers readily receive specimens that are submitted because they are suspect for a variety of diseases. The infectious nature of the specimens is usually unknown, but they have the potential to contain biological agents that may cause disease in animals and humans. Practices and procedures need to be in place that will minimise the risk of occupational exposure of employees to such pathogens. Unless suspected of containing a pathogen requiring a higher containment level, it is advisable that initial processing of all unknown specimens should be carried out as though the material contained a Group 2 pathogen. The most important aspects are to prevent percutaneous and mucous membrane exposure. Biological safety cabinets should be used for all manipulations that may generate aerosols. Class I or II are appropriate depending on the need for protection of the samples from contamination. Additionally, there should be no mouth pipetting, personal protective clothing should be worn with, in some cases, eye and respiratory protection, depending on the anticipated level of exposure. Although initial diagnostic procedures may be carried out at level 2, once a Group 3 organism has been isolated (or suspected) further work must be carried out at the higher containment level.

\section{Microbiological Safety Cabinets}

These are used at the different containment levels, as described in Section C.A. above. They are of three types:

Class I: An open-fronted cabinet designed specifically to provide operator protection and not to give protection to the work being handled.

Class II: An open-fronted safety cabinet, sometimes referred to as a laminar flow recirculating cabinet. They are designed to give operator protection as well as product protection.

Class III: These cabinets are closed with glove ports at the front and provide the highest degree of containment by complete separation of work and worker. Some cabinets have a removable glove port and are known as Class III/I cabinets, i.e. they can be used in either mode.

\section{Storage of Pathogens}

Storage of live pathogens requires appropriate containment and security to avoid risks due to breakage or unauthorised use of material. Storage facilities should be appropriately labelled to indicate the nature of the pathogens (e.g. their group) and the person(s) responsible for them. A complete inventory of the pathogens in storage should be kept up to date and available. Special care must be taken when opening glass vials of freeze-dried pathogens, as these can sometimes shatter. Care must be taken when working with liquid nitrogen.

Many of the considerations given above relate not only to human safety but also to prevention of the spread of infection to animals. In a veterinary laboratory an important responsibility is to minimise any risk of escape of pathogens to animals, either wild or domestic, in the outside community. Close communication must be maintained with the veterinary authorities.

\section{Physical and Chemical Hazards}

Laboratory work involves many manipulations that are potentially dangerous such as handling glassware and work with needles or other sharp instruments. There must be special facilities for the proper disposal of needles and other sharps. Laboratory staff should be protected from the risk of receiving a burn from hot solids or liquids. Autoclaves must be fitted with safety devices to prevent accidental opening of doors when under pressure and be regularly serviced and tested. Heat-protective gloves must be provided. Extreme cold can also be a risk, e.g. when working with liquid nitrogen; splashes on exposed skin can be very damaging. Gloves should be worn that provide insulation from cold and that are also waterproof to prevent penetration of the liquid nitrogen. Face masks and boots should also be worn when working with liquid nitrogen.

Irradiation is a serious health risk that may be present due to the use of X-ray machines or use of gamma-emitters or other sources. Equipment must be regularly serviced and tested. All use of radioactive material must be meticulously recorded. All staff must be provided with a radiation monitoring device and have annual health checks.

A wide range of chemicals is in use in veterinary laboratories, many of which may be toxic or mutagenic and some may be carcinogenic. It should be remembered that it is the dose that makes the poison. Vapours are especially hazardous and some chemicals can be absorbed by penetration of intact skin. Procedures sufficient to protect pregnant laboratory workers should be followed at all times. A list of hazardous chemicals must be maintained, and a file record kept of chemicals to which individual staff members could be exposed. Chemicals must be correctly stored in appropriate containers and at the correct temperature. Those that are very flammable must be kept in a fireproof chemical store. A record 
Human Safety in Veterinary Microbiology Laboratory

must be maintained of the purchase and use of hazardous chemicals. Disposal of some chemicals is subject to official regulation.

\section{Laboratory Animal Facilities}

Work with pathogens in laboratory animals poses special hazards. Animal rooms have to be constructed to appropriate standards and containment levels, just as laboratories. Containment in animal houses is very important because of the large amount of infectious agents that they may generate. Similar considerations also apply regarding the training of staff, protective clothing and the recording of working procedures. Special care must be taken to avoid injury to staff, e.g. through animals biting and kicking. Any such incidents should be recorded and wounds appropriately treated. There must be provision for incineration or rendering of carcasses and for the thorough cleansing and disinfection of animal rooms. The animal rooms should not only provide a suitable environment for the animals themselves but should be constructed and ventilated in such a way as to ensure comfort for the attendants.

\section{Emergency Provisions}

First-aid equipment should be readily available but stored in a location that is unlikely to be contaminated by work conducted in the laboratory. All staff should be aware of the importance of safety. There must be suitable procedures and equipment for dealing with spillages and decontamination. A record must be kept of all accidents.

There should be written procedures for dealing with emergency failure of air-handling systems, e.g. in biosafety cabinets or biocontainment rooms, which can lead to loss of containment.

Many laboratories have a staff safety committee to increase safety awareness and to discuss safety issues with management. Managers should be responsible for safety in their area of command and should not allow consideration of speed or cost of work to come before the safety of their staff. There should be an emergency procedure for calling medical assistance if required and for hospitalisation when needed. Fire alarms must be fitted and tested regularly. Each unit should designate a fire officer who conducts periodic fire drills to make staff aware of what to do and where to assemble in the event of fire. The fire officer is responsible for checking that everyone has left the building. Procedures for other natural disasters such as hurricanes and earthquakes should be in place where they present a risk. All these procedures should be written down and periodically reviewed.

\section{Transport of I nfectious Material}

Great care must be taken when preparing and packing diagnostic specimens for transport, to ensure that there is no breakage of containers or leakage of contents that could put at risk postal workers, couriers or staff at the receiving laboratory. Applicable local, national and international regulations for the transportation of dangerous goods and importation of animal pathogens must be followed.

\section{Conclusion}

High standards of laboratory safety that will ensure healthy working conditions for laboratory staff must be of the greatest priority. They can only be achieved by careful study of the principles involved followed by practical application to premises, facilities, operating procedures and hygiene. Training of all laboratory personnel must be a high priority and no personnel should be allowed to work until appropriate training has been completed and documented. There is a large published literature on all aspects of the subject, and further reading is recommended.

\section{References}

1. Acha, P. N. and Szyfres, B. (2001): Zoonoses and Communicable Diseases Common to Man and Animals, Third Edition. Pan American Health Organisation, Scientific Publication. PAHO, Washington DC, USA.

2. Advisory Committee on Dangerous Pathogens (1995): Categorisation of Biological Agents According to Hazard and Categories of Containment, Fourth Edition. Health and Safety Executive Books, P.O. Box 1999, Sudbury, Suffolk, CO10 6FS, UK. ISBN 0-7176-1038-1.

3. Barbeito, M. S., et. al.(1995): Recommended biocontainment features for research and diagnostic facilities where animal pathogens are used. Rev. sci. tech. Off. int. Epiz., 14, 873-887.

4. Bell, J. C., Palmer, S. R. and Payne, J. M. (1988): The Zoonoses. Edward Arnold, London, UK.

5. Beran, G. W. and Steele, J. H. (1994): Handbook of Zoonoses, Second Edition. Section A. (Bacterial, Rickettsial, Chlamydial and Mycotic Zoonoses) and Section B (Viral Zoonoses), CRC Press, USA.

6. Canadian Food Inspection Agency (1996): Containment Standards for Veterinary Facilities, Best M., ed. Canadian Food Inspection Agency, Ottawa, Ontario, Canada.

7. Collins, C. H. (1990): Health hazards in microbiology. In: Handbook of Laboratory Health and Safety Measures, Pal S.B., ed. Kluwer Academic Publications, Dordrecht, The Netherlands, 159-188.

8. Office of Biosafety, Laboratory Centre for Disease Control, Health and Welfare Canada (1996): Laboratory Biosafety Guidelines, Second Edition. Ministry of Supply and Services Canada, Cat. No. MR 21-2/1996.

9. Rayburn, S. R. (1990): The Foundations of Laboratory Safety (a Guide for the Biomedical Laboratory). Springer-Verlag, New York, USA.

10. Sewell, D. L. (1995): Laboratory associated infections and biosafety. Clin. Microbiol. Rev., 8, 389-405. 